\title{
Perfil antropométrico y somatotipo de regatistas del equipo preolímpico español de vela
}

\section{Anthropometric profile and somatotype of sailors of the spanish pre-olympic sailing team}

\author{
Lucía Reyes Sánchez y Vicente Morales Baños* \\ Facultad de Ciencias del Deporte San Javier (Universidad de Murcia). Campus de Excelencia Internacional Regional "Campus Mare Nostrum".
}

\begin{abstract}
Resumen: El objetivo de este trabajo fue describir las características antropométricas y somatotipo de los regatistas de diferentes clases, que formaron parte del Equipo Preolímpico Español de Vela entre los ańos 2006 y 2014. En el estudio participaron 50 regatistas del Equipo Preolímpico Español, se registraron los siguientes datos: peso, talla, pliegues cutáneos, perímetros de brazo, muslo y gemelo, diámetros del húmero, muñeca y fémur. Respecto al porcentaje graso, las chicas han estado alrededor de $16-20 \%$ y los chicos entre $10-18 \%$. El somatotipo medio de los regatistas fue mesomorfo exceptuando la clase Laser Radial que se inclinó hacia valores endomórficos. La conclusión es que existe un perfil ideal antropométrico y de somatotipo, en cada clase de barco y también dependiendo de la posición que ocupe el regatista en el barco, por lo que los regatistas que se acerquen a ese ideal tendrán más posibilidades de éxito.

Palabras clave: Antropometría, Somatotipo, Composición corporal, Vela.
\end{abstract}

Abstract: The aim of this study was to describe the anthropometric characteristics and somatotype of sailors from different classes, who were part of the Spanish Preolympic Sailing Team between the years 2006 and 2014. The study included 50 Spanish sailors from the Spanish Preolympic Sailing Team. The following data were recorded: weight, height, skin folds, circumferences of arm, thigh and calf, diameters of the humerus, femur and wrist. Regarding the fat percentage, the girls had around $16-20 \%$ and $10-18$ $\%$ boys. The mean somatotype of the sailors was mesomorph, apart from the Laser Radial class that leaned to endomorphic values. The conclusion is that there is an ideal anthropometric and somatotype profile in each class of ship listing, and it also depends on the position occupied by the sailor on the ship, hence the sailors who come to this ideal are more likely to succeed. Keywords: Anthropometry, Somatotype, Body Composition, Sailing.

\section{Introducción}

La vela es un deporte náutico, practicado con barcos que se mueven exclusivamente con la acción del viento, se compite en eventos llamados regatas y hay diferentes clases de barcos, que tienen diferentes características y formas de navegar, estas clases pueden necesitar uno o más tripulantes, que deben tener diferentes características y cualidades físicas para conseguir un buen rendimiento. Es un deporte en el que influyen muchos parámetros que no se pueden controlar, todos aquellos relacionados con la naturaleza y la meteorología, pero hay otros que sí se pueden controlar, como por ejemplo el material de los barcos, los propios atletas, preparación física, nutrición, técnica, táctica, trimado, etcétera. Todos estos aspectos controlables han sido objeto de muchos estudios en los últimos años (Allen et al, 2006).

Las clases olímpicas que participan en los Juegos Olímpicos van cambiando dependiendo del ciclo olímpico, esto lo decide la International Sailing Federation (ISAF), para los Juegos Olímpicos de Rio 2016 y Tokio 2020, las clases que competirán para mujeres son: Laser Radial y RsX como clases individuales, 470 y $49 \mathrm{er}$ para las clases dobles; para

Dirección para correspondencia [Correspodence address]: Vicente Morales Baños. Dirección: Facultad Ciencias del Deporte, Av. Argentina s/n, San Javier, Murcia (España). E-mail: vela@um.es hombres las clases individuales son: Laser Standard, Finn y RsX; las clases dobles son: 470 y 49 er. Mixto y dobles en la clase Nacra 17.

Actualmente se ha aumentado la conciencia de que el regatista debe ser un atleta y estar bien preparado físicamente para aguantar todos los días de regata, ya que las regatas del circuito olímpico suelen durar entre 5 y 6 días. En este sentido, el estudio de la composición corporal es uno de los criterios para la especialización de cualquier deporte, (Reilly, 2008, López et al, 2016, 2017) y más concretamente en vela ya que cada clase presenta una serie de exigencias, obligando a los regatistas a poseer una determinada morfología corporal (Bojsen-Moller et al, 2007)

Se ha visto que el perfil antropométrico de los regatistas de las diferentes clases influye en el resultado, (Neville et al, 2009; Polato et al, 2007) así como el somatotipo puede ser un indicador relativo del rendimiento, (Matsudo, 1996, citado en Pieter, 2010) por lo que el seguimiento de la evolución de los jóvenes deportistas es una de las principales funciones asumidas por clubes y federaciones deportivas en la actualidad, creando en diferentes deportes el perfil antropométrico de los atletas (Correa, 2008; López et al, 2013; Pieter, 2010; Muros et al, 2016). Este tipo de valoraciones permiten, no sólo el control de su salud y rendimiento, sino 
también detectar talentos deportivos para etapas posteriores de sus carreras deportivas, e incluso comparar su crecimiento con los actuales deportistas destacados de su disciplina (Pazo, 2010).

Debido a las diferentes características de los barcos, habrá diferencias muy significativas entre los requerimientos específicos de cada clase. Haciendo distinción de sexos, en las clases femeninas las atletas con más peso deberán estar en Laser Radial, con un somatotipo entre endomorfo y mesomorfo, al igual que las tripulantes de 470, aunque éstas también deberán de ser las más altas, porque se requiere más palanca, al ir de pie en el barco colgadas del trapecio. Diferenciando las patronas de 470 y las regatistas de la clase RsX, éstas últimas deberán de tener un somatotipo mesomorfo ya que gran parte del trabajo de esta clase recae sobre las extremidades superiores, las patronas de 470 serán las más delgadas y pequeñas. $\mathrm{Al}$ igual que con las femeninas, en las clases individuales masculinas podremos ordenar por tamaño, de menor a mayor de los atletas, las clases de la siguiente manera: RsX, Laser Standard y Finn. La clase Finn está hecha para regatistas muy corpulentos y pesados. $\mathrm{Y}$ en las clases dobles, por un lado se necesitará un patrón de 470 muy delgado y un tripulante de 470 alto, y por otro lado, la clase 49er será la que menos diferencia habrá entre el patrón y el tripulante.

El objetivo de este estudio es describir las características antropométricas y somatotipo de los regatistas de las diferentes clases olímpicas, crear un perfil ideal para cada clase y, que al mismo tiempo sirva de guía para regatistas jóvenes a la hora de la elección de una clase u otra, comparando sus datos con los aquí expuestos.

\section{Método}

\section{Muestra}

Se recogieron datos de 50 regatistas miembros del equipo preolímpico español entre los años 2006 y 2015, siguiendo el protocolo ISAK de medición antropométrica. Algunos de ellos tenían muchas mediciones recopiladas, sólo se incluyeron en el estudio las que tenían mejores resultados. No se obtuvieron datos de la clase 49er FX (femenina) y Nacra 17 (mixta) porque han sido recientemente incluidas entre las clases olímpicas para los próximos Juegos Olímpicos y no hay suficientes tripulaciones españolas para poder hacer un perfil ideal.

Hombres:

- 470: 4 patrones y 4 tripulantes

- 49er: 5 patrones y 6 tripulantes

- Laser Standard: 9 patrones

- Finn: 4 patrones

- Rsx: 3 patrones
Mujeres:

- 470: 4 patronas y 4 tripulantes

- Laser Radial: 4 patronas

- Rsx: 3 patronas

\section{Procedimiento}

A los sujetos se les tomó los siguientes datos: edad, peso, talla, pliegues (tricipital subescapular, supraespinoso, abdominal, muslo y gemelo), perímetros (brazo relajado, brazo contraído, muslo y gemelo) y diámetros (húmero, muñeca y fémur). A partir de estos datos se determinaron los pesos (graso, muscular, óseo y residual) y el porcentaje de los pesos, también se calculó el somatotipo.

A continuación se describen las ecuaciones utilizadas, citadas en Martínez-Sanz \& Urdampilleta-Otegui (2012):

El \% de masa grasa se calculó con la fórmula de Carter (1982) derivada de la fórmula de Yuhasz

- $\quad \%$ Graso para hombres $=0,1051 *$ Sum $6 \mathrm{pl}+2,585$

- $\quad \%$ Graso para mujeres $=0,1584^{*}$ Sum $6 \mathrm{pl}+3,58$

- $\quad$ Peso Graso = \%Graso* peso $/ 100$

La masa muscular se calculó con la ecuación de Lee (2000).

- Peso Músculo $=$ Talla $\left(0,00744^{*} \operatorname{Pr}\right.$ brazo relajado + $0,00088^{*} \operatorname{Pr}$ muslo $+0,00441^{*} \operatorname{Pr}$ pierna $)+\left(2,4^{*}\right.$ sexo $)-$ $0,048^{*}$ Edad + Etnia $+7,8$

Sexo: Mujeres $=0$; Hombres = 1; Etnia: -2 (asiáticos) 1,1 (afroamericanos) 0 (hispánicos)

- $\quad \%$ Músculo = Peso músculo*100/Peso

La masa ósea se calculó con la ecuación de Rocha (1975).

- Peso Óseo = 3,02*(Talla*D Muñeca*D Femo$\left.\mathrm{ral}^{*} 400\right)^{*} 0,712$

- \%Óseo = Peso óseo*100/Peso

La masa residual se obtuvo de restar al peso total el resto de los compartimentos corporales (Peso graso, peso músculo y peso óseo)

- Peso Residual $=$ Peso - Peso Graso - Peso Músculo Peso Óseo

- \%Residual = $100-\%$ Graso - \%Músculo - \%Óseo

Para el cálculo de los datos se usaron los siguientes instrumentos:

- Báscula con precisión de 100 gr.

- Tallímetro de pared con precisión de $1 \mathrm{~mm}$.

- LipocalibreHoltain con precisión de 0,2 mm.

- Paquímetros de diámetros óseos Holtain.

- Cinta métrica metálica, estrecha e inextensible (homologada).

- Bolígrafo para calcular los puntos anatómicos y referencias antropométricas. 
Estos datos fueron extraídos de los diferentes seguimientos que hace el Departamento de Medicina de la Real Federación Española de Vela a lo largo del proceso de selección y preparación de los diferentes regatistas durante los ciclos olímpicos de Beijing 2008, Londres 2012 y Río 2016.

\section{Análisis estadistico}

Para guardar y organizar todos estos datos se creó una hoja de datos revisada por expertos en la materia, organizando una hoja de EXCEL para hacer los cálculos relacionados con el estudio.

Todos estos datos fueron tomados por un médico del deporte que lleva trabajando más de 10 años con el Equipo de Preparación Olímpica en vela. De todos los datos se halló la media y la desviación típica para poder crear un perfil del deportista tipo de la resultante de toda la muestra, así como la creación de las tablas que se exponen en resultados para una mejor lectura de los datos.

\section{Resultados}

En las dos primeras tablas se muestran los datos antropométricos obtenidos por las diferentes clases, así como el peso y la altura, clasificados por género masculino y femenino. En ambas, las unidades de medida utilizadas son: kilogramos para el peso, centímetros para la altura y milímetros para las demás mediciones (pliegues, perímetros y diámetros).

Los datos de la Tabla 1 muestran que las regatistas más altas y pesadas son las de Laser Radial y las tripulantes de 470, éstas últimas también son las que tiene un mayor perímetro de gemelo debido a los requerimientos de su posición en el barco (navegan enganchadas al trapecio y de pie). Se puede apreciar también como las regatistas que más alto tienen el sumatorio de 6 pliegues son las de Laser Radial y las patronas de 470. Éstas presentan un porcentaje de grasa mayor que las regatistas de Rsx y las tripulantes de 470 que se puede ver en la Tabla del somatotipo de las clases femeninas (3).

Tabla 1. Perfil Antropométrico Clases Femeninas.

\begin{tabular}{|c|c|c|c|c|}
\hline & \multicolumn{2}{|c|}{ Individuales } & \multicolumn{2}{|c|}{ Dobles } \\
\hline & \multirow{2}{*}{ Laser Radial } & \multirow{2}{*}{ RsX } & \multicolumn{2}{|c|}{470} \\
\hline & & & Patrón & Tripulante \\
\hline Variable & $\mathrm{M} \pm \mathrm{DT}$ & $\mathrm{M} \pm \mathrm{DT}$ & $\mathrm{M} \pm \mathrm{DT}$ & $\mathrm{M} \pm \mathrm{DT}$ \\
\hline Peso & $65,32 \pm 4,91$ & $59,1 \pm 3$ & $59,66 \pm 5,14$ & $68,7 \pm 6,18$ \\
\hline Altura & $170,7 \pm 1,82$ & $165 \pm 1,73$ & $170 \pm 7,21$ & $174,46 \pm 4,39$ \\
\hline Sumatorio $6 \mathrm{pl}$ & $103,94 \pm 17,68$ & $82,48 \pm 14,9$ & $86,95 \pm 2,47$ & $81,6 \pm 23,52$ \\
\hline Pr braz rel & $29,72 \pm 0,67$ & $29,1 \pm 0,29$ & $27,66 \pm 0,29$ & $29,33 \pm 0,58$ \\
\hline Pr braz con & $30,9 \pm 0,47$ & $30 \pm 0,5$ & $29,36 \pm 0,32$ & $30,5 \pm 0,87$ \\
\hline Pr Muslo & $55,6 \pm 6,06$ & $49,16 \pm 1,26$ & $51,16 \pm 2,08$ & $55 \pm 2$ \\
\hline Pr Gemelo & $35,45 \pm 1,68$ & $24,33 \pm 0,29$ & $34,83 \pm 1,44$ & $36,66 \pm 0,58$ \\
\hline D Húmero & $6,42 \pm 0,15$ & $6,3 \pm 0,2$ & $6,36 \pm 0,38$ & $6,36 \pm 0,12$ \\
\hline D Muñeca & $5,1 \pm 0,18$ & $5,26 \pm 0,15$ & $5,23 \pm 0,4$ & $5,25 \pm 0,25$ \\
\hline D Femoral & $9,05 \pm 0,42$ & $9,13 \pm 0,23$ & $9,26 \pm 0,46$ & $9,43 \pm 0,21$ \\
\hline
\end{tabular}

M: media. DT: desviación típica. Sum 6pl: sumatorio 6 pliegues. Pr brazrel: Perímetro brazo relajado. Pr braz con: perímetro brazo contraido. D: diámetro.

En la Tabla 2 se aprecia que los deportistas más altos y pesados están en la clase Laser Standard y Finn, también son éstos los que muestran un mayor sumatorio de 6 pliegues y mayo- res perímetros tanto en el muslo como en el gemelo, debido a los requerimientos de sus clases, al tener que soportar todo el peso del cuerpo colgados sobre los muslos. 
Tabla 2. Perfil Antropométrico clases masculinas.

\begin{tabular}{|c|c|c|c|c|c|c|c|}
\hline & \multicolumn{3}{|c|}{ Individuales } & \multicolumn{4}{|c|}{ Colectivas } \\
\hline & \multirow{2}{*}{ Laser Standard } & \multirow{2}{*}{ Finn } & \multirow{2}{*}{ RsX } & \multicolumn{2}{|c|}{470} & \multicolumn{2}{|c|}{$49 \mathrm{er}$} \\
\hline & & & & Patrón & Tripulante & Patrón & Tripulante \\
\hline Variable & $\mathrm{M} \pm \mathrm{DT}$ & $\mathrm{M} \pm \mathrm{DT}$ & $\mathrm{M} \pm \mathrm{DT}$ & $\mathrm{M} \pm \mathrm{DT}$ & $\mathrm{M} \pm \mathrm{DT}$ & $\mathrm{M} \pm \mathrm{DT}$ & $\mathrm{M} \pm \mathrm{DT}$ \\
\hline Peso & $80,6 \pm 2,8$ & $99,1 \pm 7,3$ & $72,9 \pm 2,2$ & $66,7 \pm 1$ & $72,4 \pm 0,8$ & $72,4 \pm 3,9$ & $76,3 \pm 4,1$ \\
\hline Altura & $182,9 \pm 3,7$ & $186,8 \pm 2,7$ & $178,6 \pm 1,5$ & $175 \pm 4$ & $181,2 \pm 2,8$ & $175,7 \pm 4,6$ & $178 \pm 4,1$ \\
\hline Sum $6 \mathrm{pl}$ & $66,9 \pm 23,1$ & $88,4 \pm 17,4$ & $41,1 \pm 6,3$ & $42,4 \pm 11,5$ & $42,3 \pm 7,3$ & $53,1 \pm 7,1$ & $53,6 \pm 10,4$ \\
\hline Pr braz rel & $35,1 \pm 1,1$ & $34,7 \pm 0,3$ & $30 \pm 1,7$ & $29,8 \pm 1,6$ & $30,9 \pm 0,2$ & $33,1 \pm 1,4$ & $33,8 \pm 1,4$ \\
\hline Pr braz con & $35,8 \pm 1,9$ & $38 \pm 2,4$ & $33,2 \pm 1,9$ & $32,4 \pm 0,7$ & $34,2 \pm 0,7$ & $34,7 \pm 1,3$ & $36 \pm 1,6$ \\
\hline Pr Muslo & $58 \pm 2,3$ & $64,6 \pm 4,9$ & $53 \pm 1,7$ & $51,5 \pm 4,1$ & $52,5 \pm 2,6$ & $56,7 \pm 3,2$ & $57,4 \pm 4,8$ \\
\hline Pr Gemelo & $37,8 \pm 1,5$ & $44 \pm 2,5$ & $37,3 \pm 0,4$ & $34,8 \pm 1,2$ & $35,9 \pm 1,7$ & $36,9 \pm 1,1$ & $37,7 \pm 2$ \\
\hline D Húmero & $7,1 \pm 0,1$ & $7,5 \pm 0,1$ & $7,2 \pm 0,5$ & $7 \pm 0,2$ & $6,9 \pm 0,4$ & $6,8 \pm 0,4$ & $7,1 \pm 0,4$ \\
\hline D Muñeca & $5,9 \pm 0,3$ & $6,1 \pm 0,4$ & $5,7 \pm 0,1$ & $5,6 \pm 0,3$ & $5,5 \pm 0,3$ & $6 \pm 0,9$ & $5,8 \pm 0,1$ \\
\hline D Femoral & $10,3 \pm 0,3$ & $10,9 \pm 0,4$ & $10,2 \pm 0,2$ & $10,1 \pm 0,3$ & $10 \pm 0,1$ & $9,7 \pm 0,4$ & $9,8 \pm 0,4$ \\
\hline
\end{tabular}

M: media. DT: desviación típica. Sum 6pl: sumatorio 6 pliegues. Pr brazrel: Perímetro brazo relajado. Pr braz con: perímetro brazo contraido. D: diámetro.

En las siguientes tablas se muestran las diferencias entre clases de los pesos de los diferentes componentes del cuerpo humano, así como el porcentaje y el somatotipo de cada grupo. Los pesos se han medido en kilogramos. El somatotipo está hecho mediante la clasificación de Sheldon (1954)
El mayor porcentaje graso entre las clases, lo tienen las chicas de Laser Radial, con un somatotipo endomorfo, son los únicos datos diferentes en relación con las otras clases que tienen un porcentaje graso cerca de $16 \%$ y un somatotipo mesomorfo. (Tabla 3).

Tabla 3. Somatotipo de las clases femeninas.

\begin{tabular}{|c|c|c|c|c|}
\hline & \multicolumn{2}{|c|}{ Individuales } & \multicolumn{2}{|c|}{ Dobles } \\
\hline & \multirow{2}{*}{ Laser Radial } & \multirow{2}{*}{ RsX } & \multicolumn{2}{|c|}{470} \\
\hline & & & Patrón & Tripulante \\
\hline Variable & Media \pm DT & Media \pm DT & Media \pm DT & Media \pm DT \\
\hline Peso Graso & $12,87 \pm 2,49$ & $9,6 \pm 1,37$ & $10,11 \pm 0,71$ & $11,03 \pm 2,39$ \\
\hline Peso Músculo & $28,79 \pm 1,11$ & $27,31 \pm 2,28$ & $26,73 \pm 1,58$ & $33,43 \pm 6,29$ \\
\hline Peso Óseo & $9,99 \pm 0,21$ & $9,82 \pm 0,43$ & $10,35 \pm 1,57$ & $9,87 \pm 1$ \\
\hline Peso Residual & $13,65 \pm 0,82$ & $12,35 \pm 0,63$ & $12,47 \pm 1,07$ & $14,35 \pm 1,29$ \\
\hline$\%$ Graso & $19,59 \pm 2,74$ & $16,26 \pm 2,31$ & $16,96 \pm 0,38$ & $16,13 \pm 3,64$ \\
\hline \% Músculo & $44,15 \pm 1,88$ & $46,2 \pm 2,51$ & $44,84 \pm 0,87$ & $48,43 \pm 5,21$ \\
\hline \% Óseo & $15,35 \pm 1,14$ & $16,62 \pm 0,23$ & $17,29 \pm 1,25$ & $14,52 \pm 2,69$ \\
\hline \% Residual & $20,90 \pm 0$ & $20,9 \pm 0$ & $20,9 \pm 0$ & $20,9 \pm 0$ \\
\hline Endomorfo & $4,4 \pm 1,45$ & $3,26 \pm 0,69$ & $3,44 \pm 0,19$ & $3,2 \pm 1,16$ \\
\hline Mesomorfo & $4,01 \pm 0,53$ & $5,86 \pm 0,29$ & $3,87 \pm 0,2$ & $3,12 \pm 1,8$ \\
\hline Ectomorfo & $2,42 \pm 0,73$ & $2,43 \pm 0,27$ & $3,27 \pm 0,69$ & $2,63 \pm 0,24$ \\
\hline
\end{tabular}

M: media. DT: desviación típica. \%: porcentaje.

En la Tabla 4 se aprecia que el valor del porcentaje graso está alrededor del $10 \%$ en las clases dobles e inferior en la clase RsX, pero aumenta en la clase Laser Standard y, en mayor medida, en la clase Finn. Respecto al somatotipo todos los regatistas masculinos son mesomórficos. 
Tabla 4. Somatotipo clases masculinas.

\begin{tabular}{|c|c|c|c|c|c|c|c|}
\hline & \multicolumn{3}{|c|}{ Individuales } & \multicolumn{4}{|c|}{ Colectivas } \\
\hline & \multirow{2}{*}{ Laser Standard } & \multirow{2}{*}{ Finn } & \multirow{2}{*}{ RsX } & \multicolumn{2}{|c|}{470} & \multicolumn{2}{|c|}{$49 \mathrm{er}$} \\
\hline & & & & Patrón & Tripulante & Patrón & Tripulante \\
\hline Variable & $\mathrm{M} \pm \mathrm{DT}$ & $\mathrm{M} \pm \mathrm{DT}$ & $\mathrm{M} \pm \mathrm{DT}$ & $\mathrm{M} \pm \mathrm{DT}$ & $\mathrm{M} \pm \mathrm{DT}$ & $\mathrm{M} \pm \mathrm{DT}$ & $\mathrm{M} \pm \mathrm{DT}$ \\
\hline Peso Graso & $11,2 \pm 2,9$ & $17,2 \pm 4,1$ & $7,2 \pm 0,9$ & $6,7 \pm 1,1$ & $7,3 \pm 0,7$ & $7,5 \pm 2,1$ & $8,2 \pm 2,2$ \\
\hline Peso Músc & $39,1 \pm 3,5$ & $46,3 \pm 3,1$ & $37,8 \pm 0,8$ & $34,1 \pm 1,7$ & $37,6 \pm 1,9$ & $36,3 \pm 3,1$ & $39 \pm 2,1$ \\
\hline Peso Óseo & $13,5 \pm 0,9$ & $14,9 \pm 1,1$ & $12,6 \pm 0,1$ & $12 \pm 0,6$ & $12,4 \pm 0,8$ & $12,3 \pm 1,8$ & $12,4 \pm 0,8$ \\
\hline Peso Res & $16,0 \pm 0,6$ & $20,7 \pm 1,5$ & $15,2 \pm 0,4$ & $13,9 \pm 0,2$ & $15,1 \pm 0,2$ & $16 \pm 1,6$ & $16,7 \pm 1,2$ \\
\hline$\%$ Graso & $13,9 \pm 3,6$ & $17,2 \pm 2,7$ & $9,8 \pm 1$ & $10,1 \pm 1,8$ & $10 \pm 1,1$ & $10,4 \pm 2,6$ & $10,7 \pm 2,7$ \\
\hline \% Músculo & $48,5 \pm 4$ & $46,8 \pm 2,6$ & $51,9 \pm 0,5$ & $51,1 \pm 2,3$ & $52 \pm 2,1$ & $50,3 \pm 3$ & $51,1 \pm 1,4$ \\
\hline \% Óseo & $16,8 \pm 1,0$ & $15,1 \pm 1,6$ & $17,4 \pm 0,4$ & $18 \pm 0,7$ & $17,1 \pm 1,2$ & $17,1 \pm 2,7$ & $16,2 \pm 0,6$ \\
\hline \% Residual & $20,9 \pm 0$ & $22,5 \pm 1,8$ & $20,9 \pm 0$ & $21,1 \pm 1,4$ & $21,8 \pm 1,4$ & $22,8 \pm 1,7$ & $22 \pm 1,6$ \\
\hline Endomorfo & $3,1 \pm 0,8$ & $4,2 \pm 0,9$ & $1,7 \pm 0,3$ & $2,1 \pm 0,5$ & $2,1 \pm 0,3$ & $2,3 \pm 0,3$ & $2,3 \pm 0,6$ \\
\hline Mesomorfo & $5,3 \pm 0,6$ & $6,1 \pm 1,2$ & $5,4 \pm 0,8$ & $5,2 \pm 0,8$ & $4,6 \pm 0,5$ & $5,4 \pm 0,7$ & $5,7 \pm 0,8$ \\
\hline Ectomorfo & $2,4 \pm 0,6$ & $1,2 \pm 0,7$ & $2,7 \pm 0,6$ & $3 \pm 0,6$ & $3,2 \pm 0,6$ & $2,3 \pm 0,7$ & $2,1 \pm 0,6$ \\
\hline
\end{tabular}

M: media. DT: desviación típica. Peso Músc: peso muscularPeso Res: peso residual \%: porcentaje. Mesomorf: mesomorfo.

\section{Discusión}

Se han comparado los resultados de este estudio con las medidas estándar que Canda (2012) hizo a una muestra amplia de deportistas españoles que compitieron a nivel nacional e internacional en diferentes disciplinas deportivas y que pasaron un reconocimiento médico-deportivo en el Centro de Medicina del Deporte, perteneciente al Consejo Superior de Deportes, en los últimos diez años.

En el estudio de Canda (2012) se muestra que el peso medio de los deportistas es de 59,3 $\pm 9,04 \mathrm{~kg}$ para las mujeres $\mathrm{y}$ de 75,2 $\pm 12,8 \mathrm{~kg}$ para los hombres, por lo que las regatistas de Laser Radial y las tripulantes de 470 están por encima de esa muestra, los regatistas de la clase Finn también se encuentran muy por encima de la media de los deportistas españoles, así como, los regatistas de la clase Laser Standard también estarían por encima de la media, aunque dentro de unos valores no tan elevados.

Siguiendo esta línea, respecto la altura media de los deportistas, en el estudio comparado, la media en mujeres es de $166,3 \pm 7,39 \mathrm{~cm}$ y en hombres $179,5 \pm 8,33 \mathrm{~cm}$, tanto los regatistas de las clases masculinas como femeninas entran dentro de esos parámetros, por lo que no se aprecian datos extraordinarios. Pasa igual con los diferentes perímetros y diámetros medidos, no se aprecian datos de los regatistas muy diferentes a los registrados por la muestra de deportistas españoles de todas las disciplinas.

Para los datos del sumatorio de los 6 pliegues cutáneos, la media de los deportistas espańoles es 78,97 \pm 30,66 mm en mujeres y 53,20 $\pm 24,45 \mathrm{~mm}$ en hombres. En comparación con las regatistas femeninas, éstas están un poco por encima de la media en todas las clases, incluso en Laser Radial, que se eleva más la diferencia respecto la media. Por parte de los hombres, es en la clase Finn en la que encontramos la mayor diferencia, también por arriba de la media de la muestra de deportistas españoles.

Un dato curioso, es que comparando el estudio de Canda (2012) respecto al porcentaje graso $(18,14 \pm 5,07 \%$ en mujeres y $10,07 \pm 4,49 \%$ en hombres) en las mujeres regatistas no hay ninguna que destaque por encima de la media, aunque en hombres, son otra vez los deportistas de Finn y los de Laser Estándar, éstos últimos en menor medida, los que se encuentran por encima de la media de deportistas españoles. El porcentaje muscular medio, es de 40,4 $\pm 3,57 \%$ en mujeres y 46 $\pm 3,05 \%$ en hombres, de los deportistas españoles, es menor que la muestra de los regatistas de cualquier clase.

La media de los datos de somatotipo de los deportistas españoles, en mujeres (endomorfo: 3,4 $\pm 1,25$; mesomorfo 4,2 $\pm 1,11$; ectomorfo: $2,8 \pm 1,1$ ) y para los hombres (endomorfo: $2,3 \pm 1,08$; mesomorfo: $5,3 \pm 1,28$; ectomorfo: $2,7 \pm 1,14$ ), es un dato que se analiza de la siguiente manera: las chicas de Laser Radial, por las características del barco se ven obligadas a pesar cerca de los $70 \mathrm{~kg}$, pero miden alrededor de los $170 \mathrm{~cm}$ de altura, por lo que su somatotipo se acerca más al endomorfismo que al mesomorfismo del resto de clases en vela. Siendo los regatistas de RsX femenino y masculino los que más somatotipo mesomórfico tienen, debido a las exigencias de su clase, ya que es mucho más exigente en los miembros superiores y el tórax al ser ellos los que en todo momento triman la vela directamente desde la botavara, completamente diferen- 
te al resto de clases, que tienen una posición de sentados o de pie en el barco, y triman las velas desde las diferentes escotas.

Entre los datos obtenidos en este estudio, se comprobó que las regatistas de la clase Laser Radial y las tripulantes de 470 son las más altas y pesadas, llegando a pesar alrededor de los $70 \mathrm{~kg}$ y midiendo más de $170 \mathrm{~cm}$ de altura. En el otro extremo estarán las patronas de 470 y las chicas de RsX que pesaron menos de $60 \mathrm{~kg}$.

En las clases masculinas los chicos con menos peso se encontraron en la clase 470, siendo también los más bajos respecto a otras clases. Los chicos más altos se encontraron en Laser Standard, Finn, tripulantes de 470 y, tanto patrones como tripulantes de 49er. Siendo los chicos de la clase Finn los más pesados.

\section{Conclusiones}

El presente estudio crea un perfil antropométrico y de somatotipo de algunas de las clases olímpicas en vela.
- La mayoría de clases tienen un somatotipo mesomórfico, excepto Laser Radial, las tripulantes de 470 y los Finn.

- Son más pesados y altos los regatistas de las clases individuales (excepto los Rsx) y los tripulantes en las clases dobles.

Estos resultados pueden servir de guía para regatistas que quieran dar el salto de clases juveniles, a las clases olímpicas, sabiendo cual será la clase que más se adapte a sus características físicas.

Desde las Federaciones Autonómicas o los propios Clubes, haciendo el mismo protocolo de medición (ISAK) realizado en este estudio, podrían dirigir o aconsejar a los regatistas, para que eligieran una clase que tengan más probabilidades de éxito.

\section{Bibliografía}

1. Allen, J.B. \& De Jong, M.R. (2006). Sailing and sports medicine: a literatura review. British Journal of Sports Medicine, 40(7), 587-593.

2. Bojsen-Møller, J.; Larsson, B.; Magnusson, S.P. \& Aagaard, P. (2007). Yacht type and crew-specific differences in anthropometric, aerobic capacity, and muscle strength parameters among international Olympic class sailors. Journal of sports sciences, 25(10), 1117-1128.

3. Canda, A.S. (2012). Variables antropométricas de la población deportista española. Madrid: Consejo Superior de Deportes. Ministerio de educación, cultura y deporte.

4. Correa, J. E. (2008). Determinación del perfil antropométrico y cualidades físicas de niños futbolistas de Bogotá. Revista Ciencias de la Salud, 6(2).

5. López, G. F., Nicolás, J. \& Díaz, A. (2016). Effects of a program of intense physical activity on the body composition of adolescents from Murcia. SPORT TK: Revista EuroAmericana de Ciencias del Deporte, 5(2), 83-88.

6. López-Sánchez, G. F., Díaz-Suárez, A., Radzimiński, Ł., \& Jastrzębski, Z. (2017). Effects of a 12-week-long program of vigorous-intensity physical activity on the body composition of 10-and 11-year-old children. Journal of Human Sport \& Exercise, 12(1), 235-244. doi: 10.14198/ jhse.2017.121.19

7. López, R.; Medeiros, A.; Alfonso, J.; Batista, G.R. \& Palao, J.M. (2013). Somatotype and body composition of Brazial women beach volleyball players in relation to playing function. Medicine and Science in Sports and Exercise, 5(45), 446-446.
8. Martinez-Sanz, J.M. \& Urdampilleta-Otegui, A. (2012). Protocolo de medición antropométrica en el deportista y ecuaciones de estimaciones de la masa corporal. EFDeportes.com, Revista digital, 174.

9. Muros, J.J.; Cofre-Bolados, C.; Salvador-Pérez, S.; Castro-Sánchez, M.; Valdivia-Moral, P.; Pérez-Cortés A.J. (2016). Relación entre nivel de actividad física y composición corporal en escolares de Santiago (Chile). Journal of Sport and Health Research, 8(1):65-74.

10. Neville, V.; Calefato, J.; Pérez-Encinas, C.; Rodilla-Sala, E.; Rada-Ruiz, S.; Dorochenko, P. \& Folland, J.P. (2009). America's Cup yacht racing: race analysis and physical characteristics of the athletes. Journal of Sports Science, 27(9), 915-923.

11. Pazo, C.I. (2010) El proceso de formación de los jugadores españoles de fútbol de alta competición. Huelva.

12. Pieter, W. (2010). Detección de talentos en practicantes de Taekwondo. Revista de Artes Marciales Asiáticas, 5(2), 77-96.

13. Polato, D.; Bittencourt-Alves, R.; Carli-Arias, K.; \& Dernandes de Oliveira, L. (2007). Relation between performance and antropometric and functional profiles of optimist sailors. XXV ISBS Symposium, Our Preto - Brazil.

14. Reilly, T. (2008). The international face of sports science through the window of the Journal of Sports Sciences - with special reference to kinanthropometry. Journal of Sports Sciences, 36(4), 349-363.

15. Sheldon, W. (1954). Atlas of men: A Guide for Somatotyping the Adult Male at All Ages. New York: Harper. 